\title{
AVALIAÇÃO SENSORIAL DO SUCO DE MAÇÃ PROCESSADO COM CASCA DE ARROZ COMO COADJUVANTE DE PRENSAGEM ${ }^{1}$
}

\author{
Raul VICENZI ${ }^{2, *}$, Aldonir Barreira BILHALVA ${ }^{3}$, Rosa de Oliveira TREPTOW ${ }^{4}$
}

\begin{abstract}
RESUMO
Suco de maçã da variedade Fuji processado com casca de arroz como auxiliar na prensagem foi avaliado sensorialmente. $\mathrm{Na}$ prensagem foi utilizada pressão de trabalho de 150 e $200 \mathrm{~kg} / \mathrm{cm}^{2}$ sobre a polpa triturada e adicionado casca de arroz nas concentrações de $0 ; 1 ; 3$ e $5 \%$. Após o processamento foram efetuadas avaliações de rendimento e sensoriais por equipe de julgadores treinados. A maior concentração de casca de arroz proporcionou maior incremento no rendimento de extração do suco de maçã, superior às médias das testemunhas. Na pressão de $150 \mathrm{~kg} / \mathrm{cm}^{2}$ a testemunha obteve um rendimento de $59,91 \%$, enquanto a concentração com $5 \%$ de casca de arroz teve um rendimento de $76,09 \%$. Para a pressão de $200 \mathrm{kgf} / \mathrm{cm}^{2}$, a testemunha apresentou média de $66,52 \%$ e a concentração com $5 \%$ de casca de arroz obteve o maior rendimento de extração, média de 73,19\%. $\mathrm{Na}$ avaliação sensorial, observa-se diferença significativa entre as testemunhas e os tratamentos, especialmente quanto aos atributos cor, odor e sabor. Na qualidade geral, os sucos obtidos com o tratamento testemunha foram considerados de meIhor qualidade que aqueles obtidos com os tratamentos onde foi adicionado auxiliar de prensagem casca de arroz, indicando que este tratamento influencia negativamente na qualidade final do produto.
\end{abstract}

Palavras-chave: maçã; suco; extração; prensagem; análise sensorial; auxiliar de prensagem.

\section{SUMMARY}

SENSORIAL EVALUATION OF APPLE JUICE PROCESSED TOGETHER WITH RICE HULL AS PRESSING AID. The apple juice of the Fuji variety went through a sensorial evaluation in which it was processed together with rice hull that served as a pressing aid. The triturated pulp was pressed under a working pressure of 150 and $200 \mathrm{kgf} / \mathrm{cm}^{2}$ and rice hull was added in a concentration of $0 ; 1 ; 3$ and $5 \%$. A group of trained testers submitted the product of the process to sensory and yield evaluations. The highest concentration of rice hull provided higher increase in the yield of the apple juice extraction, higher than the average of the witnesses. Using a working pressure of $150 \mathrm{kgf} / \mathrm{cm}^{2}$ the yield obtained with the witness was of $59.91 \%$ while the $5 \%$ rice hull concentration obtained yield of $76.09 \%$, for an applied pressure of $200 \mathrm{kgf} / \mathrm{cm}^{2}$ the witness presented average of $66.52 \%$ and the concentration of $5 \%$ of rice hull obtained the higher yield of extraction, an average of $73.19 \%$. Through the sensorial evaluation significant differences can be observed between the witnesses and treatments, especially when color, odor, and flavor are considered. The general quality of pure juice was considered to have a higher quality

${ }^{1}$ Recebido para publicação em 27/01/99. Aceito para publicação em 01/10/01.

${ }^{2}$ Departamento de Biologia e Química - UNIJUI, Curso de Química Industrial de Alimentos, Caixa Postal 489, CEP 98900-000, Santa Rosa, RS. E-mail: rvicenzi@campussr.unijui.tche.br

${ }^{3}$ Departamento de Ciência e Tecnologia Agroindustrial - FAEM/ UFPEL, Caixa Postal 354, CEP: 96010-900, Pelotas, RS.

${ }_{4}^{4}$ Departamento de Ciências de Alimentos - FCD/UFPEL, e-mail: rotreptow@hot.mail.com

${ }^{*}$ A quem a correspondência deve ser enviada. than those obtained through treatments with the rice hull pressing aid. It indicates that this treatment has a negative influence on the final quality of the product.

Keywords: apple; juice; extraction; pressing; sensorial analysis; pressing aid.

\section{1 - INTRODUÇÃO}

Como acontece normalmente com as grandes produções agrícolas, existe um excedente da safra de maçã que não é comercializado no mercado in natura. Tal excedente pode chegar a $30 \%$ da produção nacional e poderia ser destinado à industrialização [5]. O Brasil produziu em 1980, 48.715 toneladas, na safra 1995/1996 produziu 544.000 toneladas de frutas, e na safra 1999/2000 produziu 967.063 toneladas, suprindo, desta maneira, grande parte das necessidades de abastecimento do mercado interno de maçãs $[4,16]$.

Dentre as frutas consideradas de clima temperado, a maçã é a mais importante, popular e melhor distribuída pelo território nacional [8]. A maçã é uma das frutas que oferecem as mais promissoras perspectivas para a industrialização, uma vez que apresenta características favoráveis e dela podem ser obtidos muitos produtos de boa aceitação [11].

A produção e a comercialização do suco de maçãs no Brasil é muito incipiente, enquanto na Europa e nos Estados Unidos, é um dos sucos mais populares, chegando a ser apontado como o segundo suco mais consumido no mundo [6]. Diversos processos podem ser usados para a produção do suco de maçã, como a prensagem, o tratamento com enzimas, a extração por centrifugação, entre outros. Um dos métodos mais utilizados, ainda é o da prensa hidráulica, embora outros tipos sejam também utilizados: prensas pneumáticas, de cesto horizontal [2], prensas contínuas de placas, correias e tipo parafuso [1].

A escolha da prensa depende de vários fatores, como: forma de operação, custos, capacidade, rendimento de extração e utilização de auxiliares de prensagem. Estas características podem ser utilizadas para melhorar o rendimento de extração e reduzir custos [1].

De acordo com HURLEY, WEY [10], quando a pressão é aplicada sobre a polpa triturada dentro de uma câmara, a falta de volume resultante da compactação, prejudica a prensagem, dificultando a extração do suco. Assim, são necessários auxiliares de prensagem para dar firmeza à polpa triturada e formar canais no interior da massa, facilitando a drenagem do suco.

Um dos auxiliares de prensagem, utilizados para o processamento de sucos de várias frutas, é representado pela casca do arroz, misturada uniformemente na 
polpa triturada na proporção de 2,5-3,25\%, p/p [7]. A casca de arroz pode ser utilizada só ou em mistura com outros materiais. Uma mistura freqüentemente utilizada é 0,5 a $1,0 \%$ de casca de arroz e $2,0 \%$ de fibras de madeira [14].

WOSIACKI et al. [17] avaliaram sensorialmente sucos de maçãs procedentes de três cultivares mais produzidas no Brasil (Gala, Golden Delicious e Fuji) e relatam que o suco da cultivar Fuji é o preferido pelo seu sabor. GONÇALVES [9], estudando a utilização do auxiliar de prensagem casca de arroz na extração de suco de maçãs para as principais variedades nacionais, verificou, através de análise sensorial que este tratamento intensifica a coloração escura e altera levemente o sabor. Para este autor, a cultivar que forneceu suco de melhor qualidade em termos de sabor foi a Fuji.

MOHR, ADAIR [12] indicam que alguns auxiliares de prensagem podem alterar fortemente o sabor característico da fruta, especialmente as fibras de madeira e a casca de arroz não tratadas. Para MOYER, AITKEN [14] é necessário muito cuidado na escolha e no uso do auxiliar de prensagem a ser utilizado para evitar a introdução de sabores e/ou odores estranhos ao suco. PATON, HAMILTON [15] não encontraram alterações na qualidade, entre sucos produzidos sem os auxiliares de prensagem e aqueles processados com diferentes auxiliares de prensagem, entre os quais casca de arroz e casca de aveia em concentrações que variaram de 0,5 a $3,0 \% \mathrm{p} / \mathrm{p}$.

Muitos trabalhos foram desenvolvidos procurando adequar qual o melhor auxiliar de prensagem e sua concentração mais adequada na extração do suco de maçãs, visando melhorar o rendimento de extração [9]. Porém, não são freqüentes os trabalhos que contemplam a análise sensorial dos sucos produzidos com o processamento de maçãs na presença de auxiliares de prensagem.

O presente trabalho teve por objetivo avaliar sensorialmente suco de maçã proveniente do processamento com casca de arroz como coadjuvante de prensagem e determinar o rendimento de extração.

\section{2 - MATERIAIS E MÉTODOS}

O experimento foi conduzido na planta-piloto do CPACT/EMBRAPA de Pelotas - RS, sendo que a análise sensorial foi realizada nos laboratórios do Departamento de Ciência dos Alimentos da Universidade Federal de Pelotas. Foram utilizadas frutas da cultivar Fuji, procedentes de pomares comerciais de Vacaria - RS. As maçãs foram armazenadas à temperatura de aproximadamente $0,5^{\circ} \mathrm{C}$ e umidade relativa de $90-95 \%$, por um período de aproximadamente oito meses. Os tratamentos analisados foram diferentes combinações de quatro concentrações de casca de arroz $(0 ; 1 ; 3$ e $5 \%)$ e duas pressões de trabalho (150 e $\left.200 \mathrm{kgf} / \mathrm{cm}^{2}\right)$.

\section{1 - Processamento}

A extração do suco foi realizada em escala de laboratório, de acordo com o processamento descrito por
GONÇALVES [9] e DE MARTIN et al. [8], demonstrado no fluxograma da Figura 1. O auxiliar de prensagem foi constituído por casca de arroz parboilizado, obtida nos engenhos de beneficiamento de Pelotas-RS. A clarificação da casca de arroz constou de três lavagens sucessivas em água fervente e posterior secagem e esterilização em estufa à temperatura de aproximadamente $120^{\circ} \mathrm{C}$.

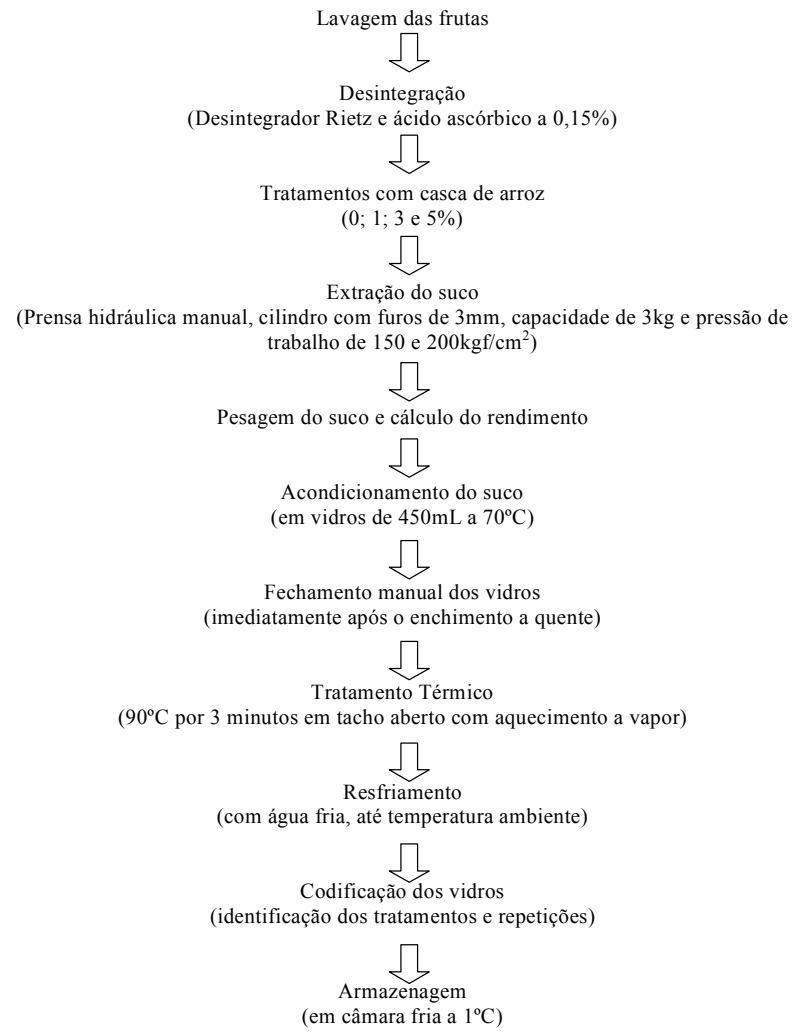

FIGURA 1. Fluxograma do processamento do suco turvo de maçã.

\section{2 - Rendimento de extração}

Obtido pela relação entre a quantidade de polpa que entrou na prensa e a quantidade de suco obtido pela prensagem. Os resultados foram expressos em percentagem. $O$ incremento no rendimento foi calculado seguindo a equação:

Incremento $(\%)=\left(n_{2}-n_{1}\right) / n_{1}$

Onde:

$\mathrm{n}_{2}$ é o rendimento obtido com auxiliar de prensagem e $\mathrm{n}_{1}$ é o valor obtido sem auxiliar de prensagem.

\section{3 - Avaliação sensorial}

$\mathrm{Na}$ seleção e treinamento de julgadores para avaliação sensorial do produto, participaram 15 pessoas de ambos os sexos, com idade variando de 20 a 45 anos. O treinamento dos julgadores quanto à definição da terminologia a ser aplicada e à diferenciação dos atributos das características, foi realizado através de 
soluções-padrões e amostras comerciais de suco de maçã, utilizando testes discriminativos e escalas não estruturadas [13], realizado em duas sessões semanais durante quatro meses. Posteriormente foram selecionados 10 julgadores que apresentaram habilidades necessárias, os quais foram testados pelo teste $F$ para cada julgador na análise de variância. Os sucos obtidos nos diferentes tratamentos foram avaliados através da aplicação de método descritivo, avaliação de atributos [3]. Os atributos avaliados nos sucos foram cor, turbidez, odor, sabor, adstringência e qualidade geral, utilizando escalas não estruturadas de $9 \mathrm{~cm}$ cujos extremos das escalas correspondem respectivamente, para cor: amarelo-claro a escuro; turbidez: não perceptível à intensa; odor e sabor característico: não perceptível a intenso; adstringência: não perceptível à intensa e à qualidade geral: péssimo a excelente.

Cada julgador recebeu quatro amostras dos tratamentos em cada sessão, com intervalos de 24 horas. As amostras foram retiradas da câmara fria e deixadas à temperatura ambiente por um intervalo não inferior a 4 horas antes das avaliações. Foram servidas em copos de vidro, codificados com três dígitos, estando os julgadores em cabines individuais. A ficha utilizada para coleta de dados está demonstrada na Figura 2.

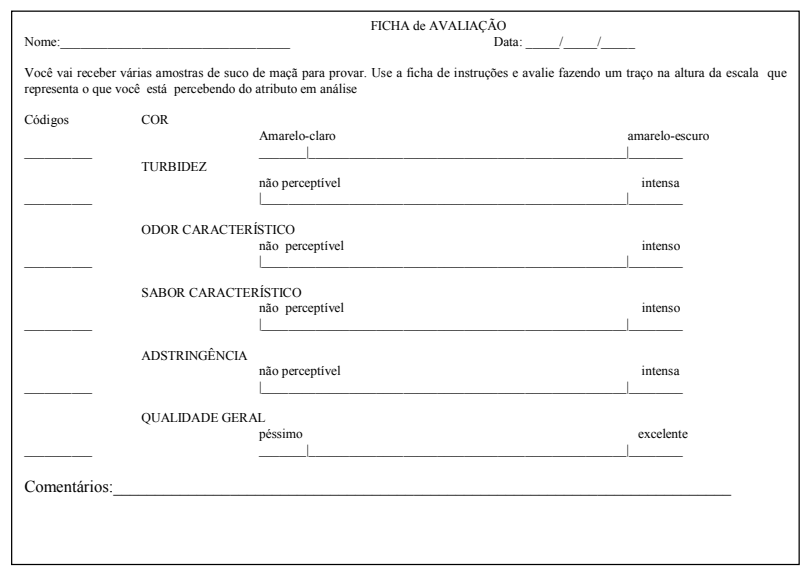

FIGURA 2. Modelo de ficha para coleta de dados na avaliação sensorial de suco de maçã.

Os dados obtidos na avaliação de rendimento foram analisados segundo o delineamento fatorial completamente casualizado com 8 tratamentos e 3 repetições, e os da avaliação sensorial foram analisados segundo o delineamento fatorial em blocos casualizados, com 10 julgadores e 8 tratamentos, através do SANEST [18].

\section{3 - RESULTADOS E DISCUSSÃO}

Observando o efeito da concentração da casca de arroz no rendimento de extração, verifica-se que há um incremento no rendimento em função do aumento da concentração da casca de arroz, para as duas pressões utilizadas. Estes resultados concordam com MOHR, ADAIR [12], PATON, HAMILTON [15] e GONÇALVES [9], que comentam a influência da adição desse auxi- liar de prensagem na extração do suco de maçã. A casca de arroz, misturada à polpa triturada de maçã, permite a formação de canais no interior da massa favorecendo o escoamento do suco, dando uma estrutura para a polpa, não permitindo a supercompactação devido à pressão aplicada e facilitando a limpeza da prensa.

Pela Tabela 1, pode-se acompanhar o incremento percentual no rendimento de extração, obtido quando se confrontam os tratamentos nos quais utilizou-se auxiliar de prensagem em diferentes concentrações e duas pressões de trabalho. Utilizando-se a testemunha (sem casca de arroz) para a pressão de $150 \mathrm{kgf} / \mathrm{cm}^{2}$ como parâmetro de avaliação, com média no rendimento de $59,91 \%$, observa-se que as concentrações 3 e $5 \%$ de casca de arroz, com médias no incremento de rendimento na ordem de 18,61 e $27,00 \%$, respectivamente, diferiram da concentração $1 \%$ de casca de arroz, com média de $12,91 \%$ no incremento de rendimento. Para a pressão de $200 \mathrm{kgf} / \mathrm{cm}^{2}$, a concentração $5 \%$ de casca de arroz, com média de incremento no rendimento de $10,02 \%$, foi significativamente superior à concentração $3 \%$, com média de $5,96 \%$, que é estatisticamente superior à concentração $1 \%$ de casca de arroz, cuja média no incremento de rendimento foi de $3,72 \%$.

Os valores encontrados para rendimento de extração se aproximam daqueles descritos por GONÇALVES [9], que utilizando pressão de $150 \mathrm{kgf} / \mathrm{cm}^{2}$ e concentração de $3 \%$ de casca de arroz, obteve um rendimento de extração na ordem de $73,50 \%$. PATON, HAMILTON [15], utilizando casca de arroz nas concentrações de 1 e $3 \%$, encontraram valores para rendimento de extração de 76,24 e 78,08\%, respectivamente. ALMASHAT, ZURITZ [1], usando $1 \%$ de casca de arroz, obtiveram um rendimento de extração de $58,50 \%$, contra um rendimento de $52,12 \%$ quando não foi usado auxiliar de prensagem, conferindo um incremento no rendimento de $12,15 \%$

TABELA 1. Incremento no rendimento de extração do suco de maçã, variedade Fuji, obtido pela prensagem com casca de arroz em três concentrações (1; 3 e $5 \%$ ) e duas pressões $\left(150\right.$ e $\left.200 \mathrm{kgf} / \mathrm{cm}^{2}\right)$.

\begin{tabular}{cccc}
\hline $\begin{array}{c}\text { Pressão } \\
\left(\mathrm{kgf} / \mathrm{cm}^{2}\right)\end{array}$ & $\begin{array}{c}\text { Concentração de casca } \\
\text { de arroz }(\%)\end{array}$ & $\begin{array}{c}\text { Rendimento } \\
(\%)\end{array}$ & $\begin{array}{c}\text { Incremento } \\
(\%)\end{array}$ \\
\hline & 0 & $59,91^{\mathrm{C}}$ & ------ \\
150 & 1 & $67,65^{\mathrm{B}}$ & $12,91^{\mathrm{B}}$ \\
& 3 & $71,06^{\mathrm{B}}$ & $18,61^{\mathrm{A}}$ \\
& 5 & $76,09^{\mathrm{A}}$ & $27,00^{\mathrm{A}}$ \\
200 & 0 & $66,52^{\mathrm{d}}$ & $---\overline{-}^{\mathrm{C}}$ \\
& 1 & $69,00^{\mathrm{c}}$ & $3,72^{\mathrm{c}}$ \\
& 3 & $70,49^{\mathrm{b}}$ & $5,96^{\mathrm{b}}$ \\
& 5 & $73,19^{\mathrm{a}}$ & $10,02^{\mathrm{a}}$ \\
\hline
\end{tabular}

Os valores refletem a média de três repetições. Médias seguidas de letras distintas na mesma coluna, maiúscula para a pressão de $150 \mathrm{kgf} / \mathrm{cm}^{2}$ e minúsculas para a pressão $200 \mathrm{kgf} / \mathrm{cm}^{2}$, indicam diferenças pelo teste de Duncan a $5 \%$.

A Tabela 2 mostra os resultados para os vários atributos sensoriais estudados. Em relação à cor sensorial, percebe-se que a concentração da casca de arroz alterou significativamente a coloração do suco. Observa-se que os tratamentos aplicados à polpa apresentam diferenças significativas. Na pressão de $150 \mathrm{kgf} / \mathrm{cm}^{2}$, onde a testemunha diferiu dos demais tratamentos, a concentração $5 \%$ de casca de arroz apresentou média 
de 6,00, sendo a coloração amarela mais escura. Para a pressão de $200 \mathrm{kgf} / \mathrm{cm}^{2}$, as colorações amarelas mais escuras foram conferidas pelas concentrações 1 e $3 \%$ de casca de arroz, enquanto a testemunha apresentou média de 1,90, diferindo das demais.

Estes valores diferem daqueles encontrados por GONÇALVES [9], que trabalhando com casca de arroz como auxiliar de prensagem, para a cultivar Fuji, encontrou média igual a 9, classificada na escala como amarelo-escuro.

Observa-se que a turbidez dos sucos foi alterada com a presença de casca de arroz na prensagem. Nota-se um ligeiro incremento na intensidade da turbidez dos sucos com o aumento da concentração de casca de arroz. A turbidez mais intensa, para a pressão de $150 \mathrm{kgf} / \mathrm{cm}^{2}$ foi conferida pela concentração $5 \%$ de casca de arroz, diferindo estatisticamente dos demais tratamentos e, para a pressão $200 \mathrm{kgf} / \mathrm{cm}^{2}$, a testemunha diferiu estatisticamente dos demais tratamentos, os quais conferiram maior intensidade na turbidez, apresentando valores correspondentes na escala.

Para a avaliação do odor característico, observase que os tratamentos aplicados à polpa para auxiliar na extração do suco, influenciaram na intensidade do odor característico do suco. Observa-se que há uma relação negativa entre a concentração de casca de arroz e intensidade do odor característico.

As testemunhas conferiram o odor característico mais intenso, diferindo dos demais tratamentos, e nas duas pressões utilizadas, a concentração $1 \%$ de casca de arroz, embora diferindo da testemunha, provocou pouca alteração no odor característico do suco, diferindo das demais concentrações de casca de arroz, as quais apresentaram, entre si, valores correspondentes na escala.

TABELA 2. Valores médios da cor sensorial, turbidez sensorial, odor sensorial, adstringência sensorial, sabor sensorial e qualidade geral no suco de maçã, cultivar Fuji, obtido com a adição do auxiliar de prensagem casca de arroz*.

\begin{tabular}{|c|c|c|c|c|c|c|c|}
\hline \multicolumn{2}{|c|}{$\begin{array}{l}\text { Tratamentos aplicados à } \\
\text { polpa na extração do suco }\end{array}$} & \multirow{2}{*}{$\begin{array}{c}\begin{array}{c}\text { Cor } \\
\text { sensorial }\end{array} \\
\text { Média }\end{array}$} & \multirow{2}{*}{$\begin{array}{c}\begin{array}{c}\text { Turbidez } \\
\text { sensorial }\end{array} \\
\text { Média }\end{array}$} & \multirow{2}{*}{$\begin{array}{c}\begin{array}{c}\text { Odor } \\
\text { sensorial }\end{array} \\
\text { Média }\end{array}$} & \multirow{2}{*}{$\begin{array}{c}\begin{array}{c}\text { Adstringência } \\
\text { sensorial }\end{array} \\
\text { Média }\end{array}$} & \multirow{2}{*}{$\begin{array}{c}\begin{array}{c}\text { Sabor } \\
\text { sensorial }\end{array} \\
\text { Média }\end{array}$} & \multirow{2}{*}{$\begin{array}{c}\text { Qualidade } \\
\text { geral } \\
\text { Média }\end{array}$} \\
\hline $\begin{array}{l}\text { Pressão } \\
\left(\mathrm{kgf} / \mathrm{cm}^{2}\right)\end{array}$ & $\begin{array}{c}\text { Casca de arroz } \\
(\%)\end{array}$ & & & & & & \\
\hline \multirow{5}{*}{150} & 0 & $1,66^{6^{d^{2}}}$ & $3,22 \mathrm{~d}^{\mathrm{d} m}$ & $6,66^{\mathrm{a}^{* *}}$ & $0,68^{\mathrm{an}^{* *}}$ & $7,33^{\mathrm{a}^{x^{*}}}$ & $7,87^{\mathrm{a}^{\mathrm{a}^{*}}}$ \\
\hline & 1 & $4,25^{\circ}$ & $5,93^{\mathrm{c}}$ & $6,17^{b}$ & $0,50^{a}$ & $6,33^{\mathrm{b}}$ & $6,21^{b}$ \\
\hline & 3 & $5,12^{\mathrm{b}}$ & $6,41^{\mathrm{b}}$ & $4,81^{\circ}$ & $0,50^{\mathrm{a}}$ & $6,01^{\mathrm{b}}$ & $6,33^{\mathrm{b}}$ \\
\hline & 5 & $6,00^{\mathrm{a}}$ & $7,55^{\mathrm{a}}$ & $4,37^{\circ}$ & $0,45^{\mathrm{a}}$ & $5,92^{\mathrm{c}}$ & $5,69^{\circ}$ \\
\hline & 0 & $1,90^{c}$ & $4,38^{\mathrm{b}}$ & $6,67^{\mathrm{a}}$ & $0,54^{\mathrm{a}}$ & $7,13^{\mathrm{a}}$ & $7,17^{\mathrm{a}}$ \\
\hline \multirow{3}{*}{200} & 1 & $4,39^{\mathrm{a}}$ & $6,07^{\mathrm{a}}$ & $6,10^{\mathrm{b}}$ & $0,36^{\mathrm{a}}$ & $6,55^{\mathrm{b}}$ & $6,47^{\mathrm{b}}$ \\
\hline & 3 & $4,52^{\mathrm{a}}$ & $6,27^{\mathrm{a}}$ & $5,36^{\mathrm{C}}$ & $0,46^{\mathrm{a}}$ & $6,35^{\mathrm{b}}$ & $6,57^{\mathrm{b}}$ \\
\hline & 5 & $2,71^{\mathrm{b}}$ & $5,16^{\mathrm{a}}$ & $4,84^{\mathrm{d}}$ & $0,45^{\mathrm{a}}$ & $4,50^{\circ}$ & $5,16^{\mathrm{c}}$ \\
\hline
\end{tabular}

* Valores médios de dez repetições. Letras distintas, na mesma coluna, indicam diferenças estatísticas pelo teste de Duncan a $5 \%$.

** Os menores valores correspondem à cor amarela mais clara; menor intensidade de turbidez; odor e sabor característicos menos perceptíveis; adstringência menos perceptível e menor qualidade geral dos sucos.

Para a adstringência, observa-se que não há variação entre as testemunhas e os tratamentos utilizados, ficando os valores próximos ao extremo inferior da escala, que variou de adstringência não perceptível à intensa.
Os valores estão em sintonia com aqueles encontrados por GONÇALVES [9], onde os tratamentos com casca de arroz não diferiram da testemunha.

Para a análise sensorial do sabor característico, observa-se uma maior perda da intensidade do sabor característico com o aumento na concentração de casca de arroz na prensagem. O menor valor obtido, indicando uma grande perda na intensidade do sabor característico, foi quando utilizou a máxima pressão e concentração de $5 \%$ de casca de arroz, não havendo diferença entre a concentrações 1 e $3 \%$ de casca de arroz, cujas médias foram correspondentes na escala, nas duas pressões utilizadas.

Há uma relação direta entre a alteração no sabor característico e o aumento na concentração de casca de arroz e aumento da pressão utilizada. As testemunhas foram significativamente superiores aos tratamentos quanto ao sabor característico do suco e não houve diferenças significativas entre as médias obtidas com as concentrações 1 e $3 \%$ de casca de arroz.

Os resultados, encontrados para o atributo sabor característico, concordam com GONÇALVES [9], que verificou alteração de sabor no suco em função do emprego da casca de arroz utilizada como auxiliar de prensagem na extração do suco de maçã. MOHR, ADAIR [12] relatam que a casca de arroz como auxiliar de prensagem pode alterar fortemente o sabor do suco. Porém, discordam de PATON, HAMILTON [15], os quais não encontraram alteração no sabor do suco de maçã com a utilização da casca de arroz.

$\mathrm{Na}$ avaliação sensorial da qualidade geral do suco, verifica-se que houve diferenças significativas em função do emprego da casca de arroz na extração do suco. Os menores valores, correspondendo à pior qualidade, foram obtidos pelos sucos provenientes do emprego de maior percentagem de casca de arroz no processo de extração. Nota-se, também, que não ocorrem alterações em função da utilização de diferentes pressões de trabalho.

\section{4 - CONCLUSÕES}

A utilização da casca de arroz como auxiliar de prensagem incrementa o rendimento de extração do suco de maçã da variedade Fuji.

As características sensoriais do suco de maçã são alteradas com a adição da casca de arroz na prensagem, sendo que a maior concentração de casca de arroz proporciona maiores alterações. Dentre os atributos sensoriais estudados somente a adstringência não foi alterada pela adição da casca de arroz na prensagem.

\section{5 - REFERÊNCIAS BIBLIOGRÁFICAS}

[1] AL-MASHAT, S. H. I. \& ZURITZ, C. A. Stress relaxation behavior of apple pomace and effect of temperature, pressing aid and campaction rate on juice yield. J. Food Eng. v. 20, n. 3, p. 247 266, 1993.

[2] APRIA - Association Pour la Promotion Industria Agriculture. Industrie de Jus de Fruits. Paris, Tomo 2, 1971. 
[3] ASSOCIAÇÃO BRASILEIRA DE NORMAS TÉCNICAS - NBR 12994. Métodos de Análise de Alimentos e Bebidas Classificação. São Paulo: ABNT. 2p.

[4] ASSOCIAÇÃO BRASILEIRA DE PRODUTORES DE MAÇÃ. ABPM - Estatísticas, 2000.

[5] ASSOCIAÇÃO GAÚCHA DE PRODUTORES DE MAÇÃ E PERA. J. Agapomi, n. 50, p. 4.1989

[6] BERASAIN, J. M. Aproveitamento industrial dos refugos da produção de maçã. Boletim do CEPPA, v. 4, n. 20 , p. 8-24, 1986.

[7] BINKLEY, C.R. \& WILEY, R. C. Continous diffusion extraction methods produce apple juice. J. Food Science., v. 43, p. 1019-1023, 1978.

[8] DE MARTIN, Z. J.; NISIDA, A. L.; CAMPOS, S. D.; SOLER, M.P.; BLEINROTH, E.W. Estudo da estabilidade do suco turvo de maçã das cultivares Golden Delicious e Fuji. Boletim do ITAL, v. 23, n. 3, p. 311-323, 1986.

[9] GONÇALVES, C. A. D. Extração de suco de maçãs (Malus domestica, Borkh) e suas qualidades sensoriais. Pelotas, 1992. 82p. (Dissertação de Mestrado) - Faculdade de Agronomia "Eliseu Maciel", Universidade Federal de Pelotas (UFPeL).

[10] HURLER, A. \& WEY, R. Technique of griding and making the mash. Confructa stud. v. 28, n. 2, p. 125-130, 1984.

[11] LOPEZ, A. \& CAROL, D. E. New apple products. Food Techn.. v. $19(9): 85-86,1965$.
[12] MOHR, W. P. \& ADAIR, R. G. Juice extraction studies with apple. Processing Laboratory, Smithfield Experimental Station, Research Branch, Agriculture Canada, Smithfield, Ont., 1979, 21p. (Bulletin, 1).

[13] MORAES, M. A. C. Métodos para avaliação sensorial de alimentos. Campinas: UNICAMP, 1985. 85 p.

[14] MOYER, J. C. \& AITKEN, H. C. Apple Juice. In: TRESSLER \& JOSLYN, D.K. (Ed.) Fruit and vegetable juice processing technology. Westport, AVI, 1971. p. 186233.

[15] PATON, D. \& HAMILTON, A. H. An assessment of oat hulls as a press aid for the apple juice processing industry. J. Inst. Can. Sci. Technol. Aliment. v. 21, p. 76-79, 1988.

[16] SOZO, J. Estudo sobre a cultura da maçã no Rio Grande do Sul e perspectivas de crescimento. Vacaria-RS, AGAPOMI, s.d. $28 \mathrm{p}$

[17] WOSIACKI, G.; OLIVEIRA, C. E.; SICHIRI, V. F.S.; CERIBELI, M.I.P.S.; SATUQUE, E.E.; NAMIUCHI, N. N. Avaliação do uso da maçã nacional. Parte I: Características de qualidade dos sucos das variedades Fuji, Gala e Golden Delicious. Boletim da SBCTA. v. 21, n. 2/3, p. 65-78, 1987.

[18] ZONTA, E. P. \& MACHADO, A. A. SANEST - Sistema de análise estatística para microcomputadores. Pelotas, 1984 (Registro na Secretaria Especial de Informática, sob o $\mathrm{n}^{\circ} 066060$ / cat. A). 